\title{
Planar Imaging
}

National Cancer Institute

\section{Source}

National Cancer Institute. Planar Imaging. NCl Thesaurus. Code C94954.

Implementation of a gamma camera to obtain 2D images with no tomographic reconstruction process being involved. 